PoS $\quad \begin{aligned} & \text { PROCEEDINGS } \\ & \text { OF SCIENCE }\end{aligned}$

\title{
Status of NEXT and prospects for a future Next Array apparatUS with Improved CApAbilities (NAUSICAA)
}

\author{
J.J. Gomez-Cadenas* $*$ \\ IFIC (CSIC-UV) \\ E-mail: gomez@mail.cern.ch
}

In this paper I review the NEXT project, including preliminary results from NEW (a 10-kg radiopure detector currently operating at the Canfranc Underground Laboratory), the status of NEXT-100, and the conceptual ideas of the next stage, the Next Array apparatUS with Improved CApAbilities (NAUSICAA).

XVII International Workshop on Neutrino Telescopes 13-17 March 2017

Venezia, Italy

* Speaker.

${ }^{\dagger}$ NEXT collaboration. 


\section{Introduction}

Neutrinoless double beta decay (DBD) is a hypothetical, very slow radioactive process in which two neutrons undergo $\beta$-decay simultaneously and without the emission of neutrinos. An unambiguous observation would establish that neutrinos are Majorana particles, identical to their antiparticles and would have deep implications in physics and cosmology.

During the last decade several experiments deploying different technologies have searched or are about to start searching $\beta \beta 0 v$ processes [1, 2, 3]. Three of them (GERDA[4], EXO [5] and KamLAND-Zen [6]) have recently published the results of their analysis, extending the sensitivity of previous searches by more than one order of magnitude. Two other experiments have recently started their experimental program underground. CUORE [7], is taking data with the full detector, while NEXT, a high-pressure gas TPC (HPGXe) with electroluminescent amplification [8, 9, 10] is operating the first phase of the experiment, a 10-kg demonstrator called NEW and plans to deploy the second phase (a $100 \mathrm{~kg}$ TPC called NEXT-100) in 2019. CUORE and NEXT project a sensitivity to the half-life of ${ }^{128} \mathrm{Te}\left({ }^{136} \mathrm{Xe}\right)$ in the range of $10^{26} \mathrm{yr}$.

The next step for the field is to push the current limits on DBD searches by one order of magnitude, allowing a discovery if the $\beta \beta 0 \mathrm{v}$ process half-life is of the order of $10^{27} \mathrm{y}$. In order to achieve this goal it is necessary to build detectors with target masses in the multi-ton range and extremely low background level, in the vicinity of one event per ton per year. This is an extremely challenging goal that requires pushing the existing technologies to the limit.

HPGXe TPCs appear specially well suited to spearhead the next generation of DBD searches, given the excellent energy resolution [11] and the availability of a topological signature [12] capable of providing strong background rejection. On the other hand, in order to increase the target mass by one order of magnitude, the background in the region of interest (ROI) must also be reduced by at least the same factor. This in turn requires: a) increasing the background rejection, chiefly by improving the topological signal and $\mathrm{b}$ ) reducing the radioactive budget of the detector.

In this paper I review the NEXT project, including preliminary results from NEW, the status of NEXT-100, and the conceptual ideas of the planned next stage, the Next Array apparatus with Improved CApAbilities (NAUSICAA). The discussion about the potential of the HPGXe-EL technology for Ba-tagging is presented elsewhere [13].

\section{HPGXe-EL: principle of operation and advantages}

The Neutrino Experiment with a Xenon TPC is a program to search for $\beta \beta 0 v$ processes using high pressure gas xenon TPCs with electroluminescent amplification (HPGXe-EL). The detection process, illustrated, in figure 1, is as follows. A charged particle propagating in the gas deposits its energy through both scintillation and ionization of the gas molecules. The scintillation light (UV photons at $\sim 172 \mathrm{~nm}$ ) is registered by photomultipliers (PMTs) on the cathode side of the TPC and gives the starting time of the event. The ionization electrons are drifted by an electric field all the way through the drift region until they enter a small region of moderately higher field where they are accelerated and secondary scintillation (but not ionization) occurs. This process, called electroluminescence (EL), results in an amplification of the signal, which grows linearly with the electric field as long as its magnitude remains below the ionization threshold. PMTs detect the EL 


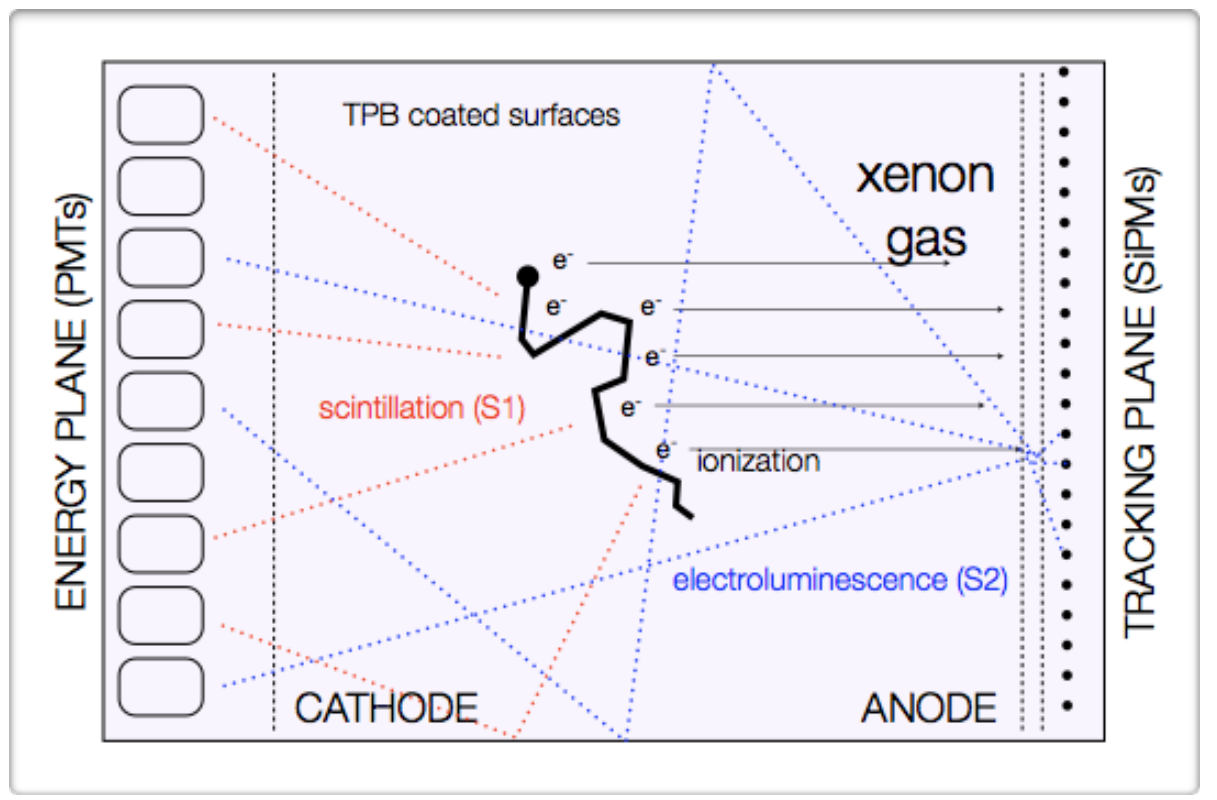

Figure 1: Principle of operation of an HPGXe-EL.

light, giving a precise measurement of the energy of the event. On the anode side, the distribution of the EL light on a grid of silicon photomultipliers (SiPMs) placed behind the EL area is a 2D picture of the track at a given position along the axis, every microsecond. Knowing the starting time of the event, the absolute position along the TPC axis can also be reconstructed.

\section{NEW and NEXT-100}

\subsection{NEW}

The NEXT-White ${ }^{1}$ (NEW) detector is a prototype of $\sim 50 \mathrm{~cm}$ of drift length and $\sim 50 \mathrm{~cm}$ diameter, which contains about $5 \mathrm{~kg}$ of xenon mass in the active volume at 15 bar. Its purpose is to understand the technology choices and background budget for the subsequent stages of NEXT, in particular the NEXT-100 detector.

The apparatus, shown schematically in figure 2 consist of a cylindrical stainless-steel vessel, designed to withstand more than 20 bar of pressure. Inside the vessel a $6-\mathrm{cm}$ thick radiopure copper shield protects the active volume from the radiation coming from the outside, including the vessel itself. The readout planes are mounted in 12-cm thick copper plates, and consist of 12 Hamamatsu R11410-10 photomultipliers (PMTs) and 1792 SensL C series silicon photomultipliers (SiPMs). The PMTs are 3-inch size and radiopure, with good quantum efficiency in the VUV and blue regions. The resulting photocathode coverage of the energy plane is about $30 \%$. Since the PMTs cannot withstand high pressure, the volume containing them is separated from the rest of the detector and maintained in an atmosphere of nitrogen at 1 bar. The PMTs are coupled to the field cage via sapphire windows coated with TPB, to shift the VUV light emitted by xenon to blue to have a better transmission through the windows. The SiPMs of the tracking plane are mounted on

\footnotetext{
${ }^{1}$ Named after Prof. James White, our late mentor and friend.
} 


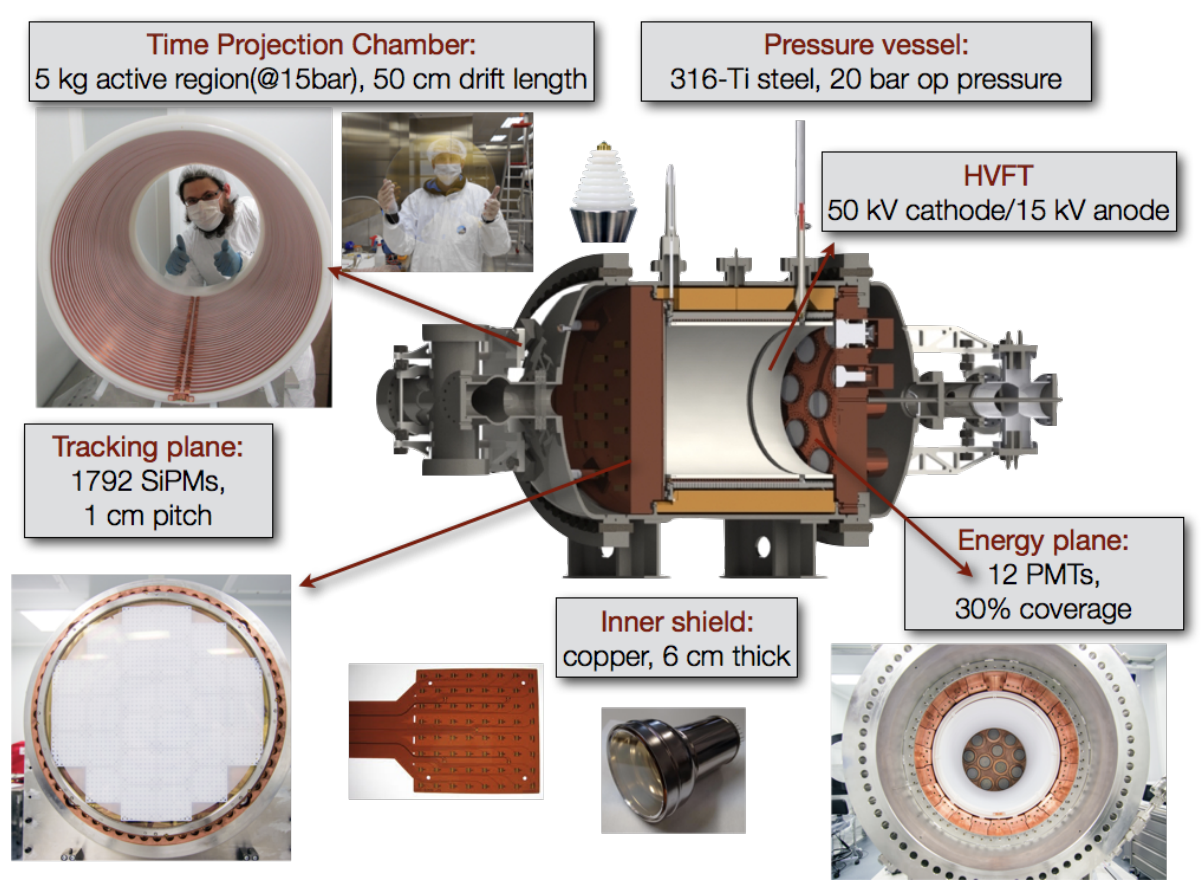

Figure 2: The NEW apparatus.

28 Kapton boards, each with $8 \times 8$ sensors spaced $1 \mathrm{~cm}$ and covered with a reflective teflon layer to improve the light collection on the PMTs. The plane is positioned behind a 3-mm thick quartz plate coated with a conductive Indium Tin Oxide layer, which constitutes the anode. The quartz plate is also coated with TPB, since SiPMs are not sensitive to VUV light. The plate defines the ground end of the EL region, the other end being a stainless steel mesh kept at negative voltage. The field cage is made of a high density polyethylene tube, which holds copper rings which maintain the uniformity of the electric field. Inside the rings, a $1-\mathrm{cm}$ thick teflon cylinder, coated with TPB serves as a reflector of light. The NEW field cage creates a homogeneous electric field of 300 $\mathrm{V} / \mathrm{cm}$ in the active volume, and a field of $2-3 \mathrm{kV} / \mathrm{cm} / \mathrm{bar}$ in the EL region. Between the cathode and the PMT plane, a buffer region degrades the high-voltage at the cathode, consisting of a series of grooves to prevent the movement of charge across the surface

The vessel is mounted on a seismic pedestal and surrounded by a lead castle $(20 \mathrm{~cm}$ thick) that shields the detector against the high-energy gamma flux from the rocks of the laboratory.

The detector has been running since October 2016 with depleted xenon, showing a high operation stability. Calibrations with Krypton X-rays as well as with other radioactive sources have been performed. Figure 3 shows preliminary results from NEW, illustrating both the excellent energy resolution and the strong topological signature characteristic of the technology. The detector will operate until the commissioning of the NEXT-100 apparatus. During 2018, a measurement of the $\beta \beta 2 v$ mode will be performed.

\subsection{NEXT-100}

Figure 4 shows a longitudinal cross-section schematic of NEXT-100. The detector is, in essence, a 2:1 replica of NEW, with an active volume of $1.15 \mathrm{~m}^{3}$. The energy plane of NEXT-100 

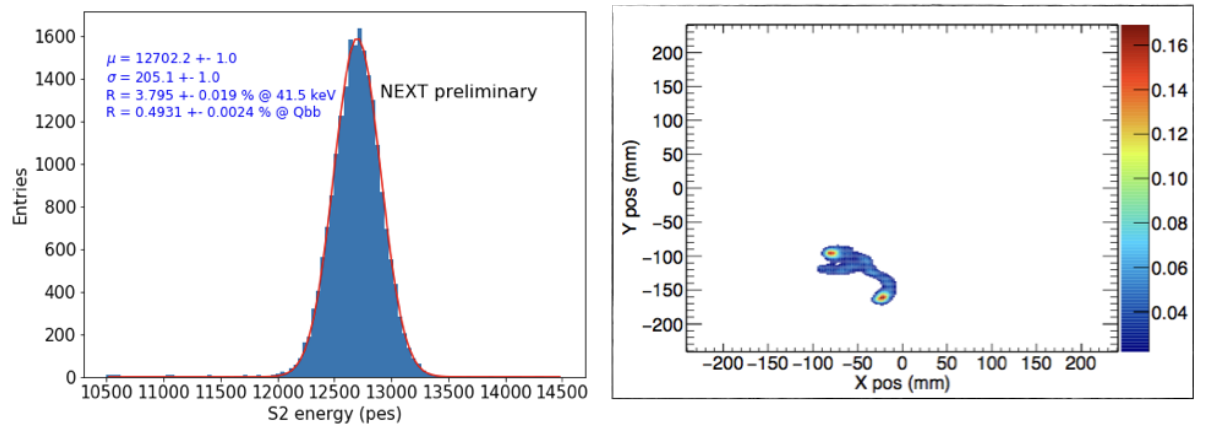

Figure 3: Preliminary results from NEW calibrations with radioactive sources. Left panel, energy resolution measured with Krypton X-rays extrapolates to better than 0.5\% FWHM; right, reconstruction of a double electron track from ${ }^{208} \mathrm{Tl}$ double escape peak, showing the characteristic two blobs.

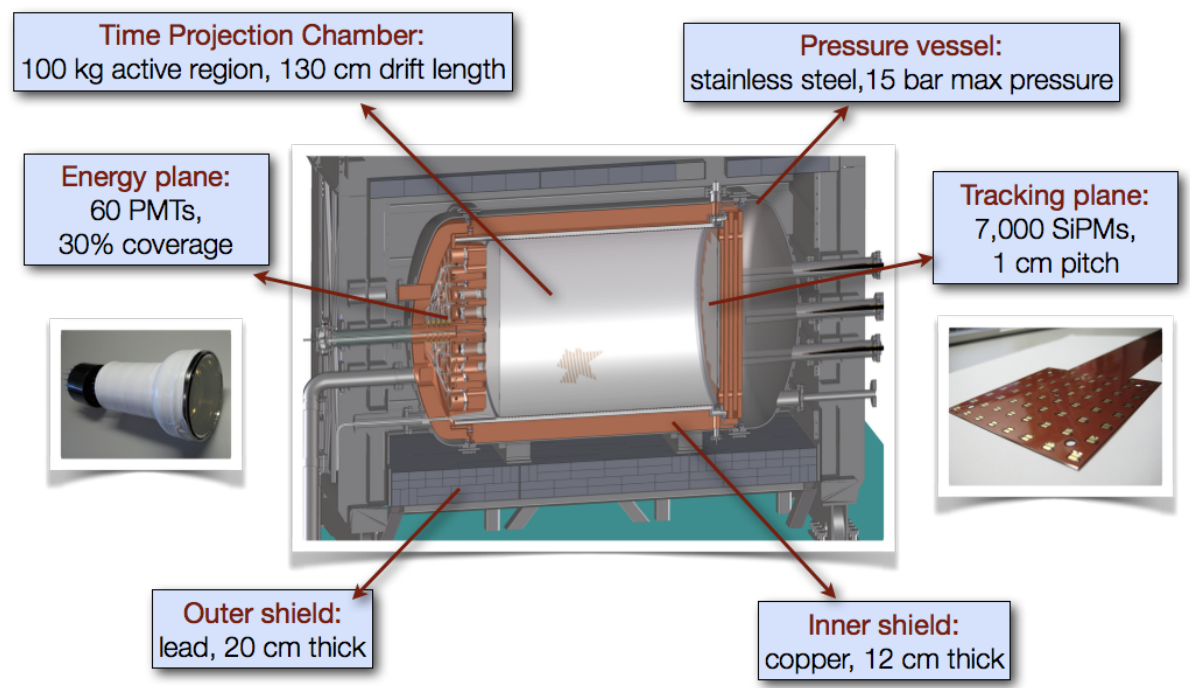

Figure 4: Cross-section view of the NEXT-100 detector inside its lead castle shield. A stainless-steel pressure vessel houses the electric-field cage and the two sensor planes (energy plane and tracking plane) located at opposite ends of the chamber. The active volume is shielded from external radiation by at least 12 $\mathrm{cm}$ of copper in all directions.

will be composed of 60 Hamamatsu R11410-10 photomultiplier tubes located behind the cathode of the TPC and covering approximately $30 \%$ of its area. The tracking plane deploys an array of $7168 \mathrm{SiPMs}$ regularly positioned at a pitch of $1 \mathrm{~cm}$ and located behind the fused-silica window that closes the EL gap. The detector will hold about $100 \mathrm{~kg}$ of xenon gas at $15 \mathrm{bar}$.

The leading background sources in NEXT are high-energy photons emitted by ${ }^{208} \mathrm{Tl}$ and ${ }^{214} \mathrm{Bi}$ contaminations, producing single electrons in the chamber with energies close to $Q_{\beta \beta}$. The ability to define a fiducial volume well separated from the detector surfaces and the capability to reconstruct tracks permits the suppression of superficial backgrounds (such as those induced by alpha particles) to negligible levels. Energy resolution results in a further suppression, in particular of the photoelectric peak of ${ }^{214} \mathrm{Bi}$ at energies very near to $Q_{\beta \beta}$. Last but not least, the topological signature (figure 5) provides a rejection of an additional order of magnitude . 

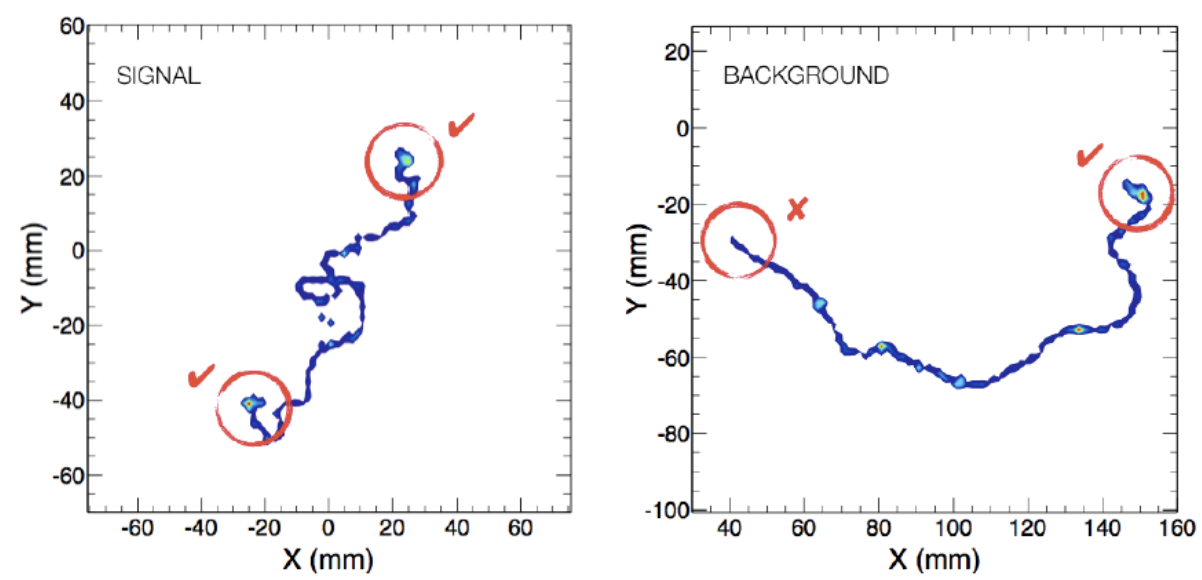

Figure 5: , simulation of a double-electron produced in a ${ }^{136} \mathrm{Xe}$ decay propagating in an HPGXe-EL; right, a single electron, arising from a high energy gamma. Right: The energy of the "blobs" found in the track extrema for signal (left) and background (right).
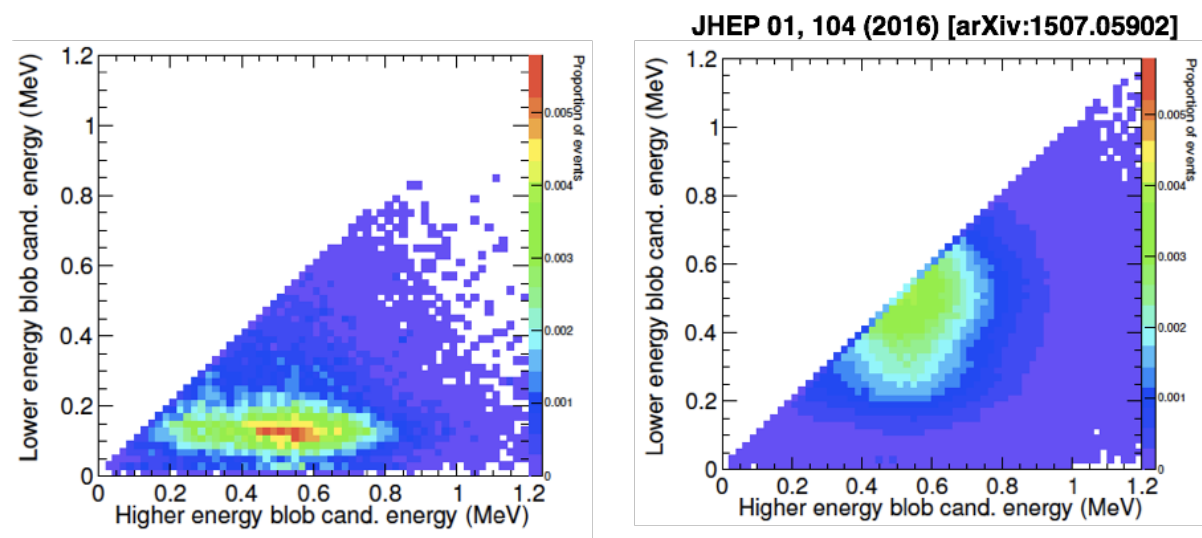

Figure 6: The topological signature of NEXT in action. The panels plot the energy of blob-1 versus blob-2, where the blobs are defined as the energy concentrations at the extrema of the track. The left panel shows the case of high-energy background electrons from ${ }^{214} \mathrm{Bi}$ and ${ }^{208} \mathrm{Tl}$ contaminations. The right panel shows the case for double electrons emitted in a $\beta \beta 0 v$ decay.

The background rate expected is $b \sim 4 \cdot 10^{-4}$ ckky [14]. Assuming a conservative resolution of $0.7 \%$ FWHM, the background in the ROI $(17.5 \mathrm{keV})$ is expected to be about 0.7 count per 100 $\mathrm{kg}$ and per year. Thus, the sensitivity of NEXT-100 increases quickly with the exposure, since the detector, at this mass scale operates as a virtually "background free experiment". A sensitivity of $10^{26} \mathrm{y}$ in $T_{1 / 2}^{0 v}$ is expected for an exposure of $500 \mathrm{~kg}$.

\section{Extrapolation of the HPGXe-EL technology to the ton scale}

The background rate of NEXT-100 extrapolates to roughly 7 counts per ton in the ROI. With such a background level, the full coverage of the inverse hierarchy would require a large exposure. Thus, at least one order of magnitude improvement in background reduction is needed to extrapolate the HPGXe-EL technology to the ton scale. 

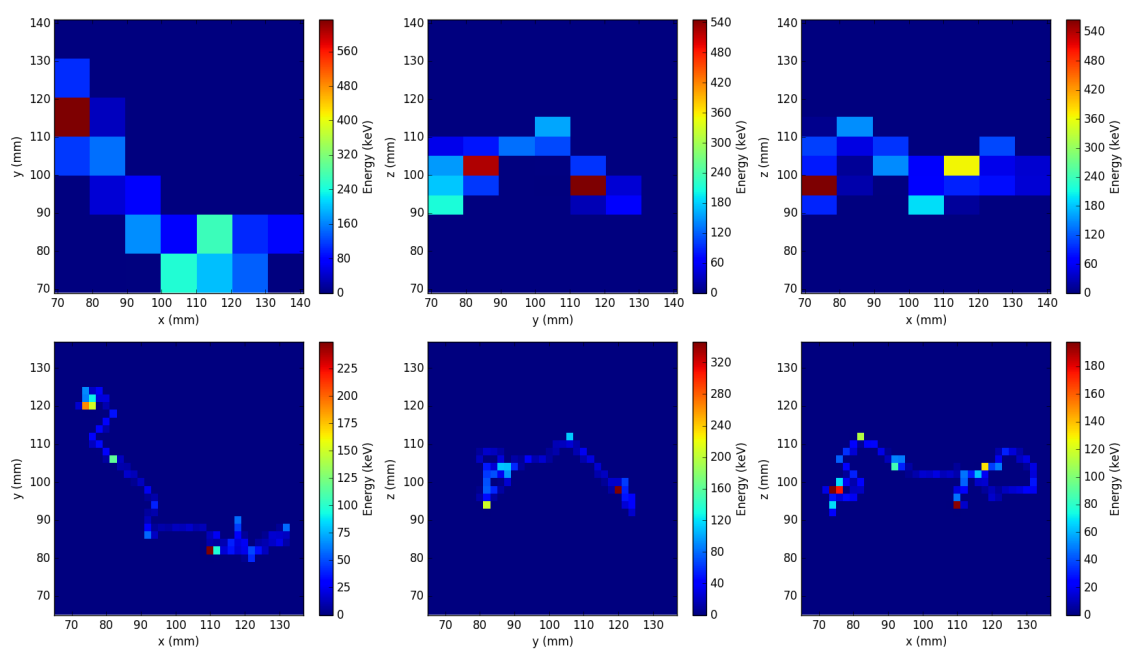

Figure 7: Projections in $x y, y z$, and $x z$ for an example signal event voxelized with $10 \times 10 \times 5 \mathrm{~mm}^{3}$ voxels (above) and with $2 \times 2 \times 2 \mathrm{~mm}^{3}$ voxels (below).

Such a background reduction may come from several sources. First, improving the energy resolution, which is assumed to be $0.7 \%$ FWHM at $Q_{\beta \beta}$ in the NEXT sensitivity calculations, while the results of the NEXT prototypes and NEW suggest that $0.5 \%$ FWHM is attainable. Second, reducing the radioactive budget, suppressing the dominant sources of background, which in the case of NEXT-100 are the PMTs and the SiPM circuit boards. The residual background from the copper shield can also be reduced by using electro-formed copper for the 1-2 $\mathrm{cm}$ of the shield closer to the gas. Third, exploiting the economy of scale, which dictates that the signal-to-noise of a detector where the signal occurs in the volume while the backgrounds accumulate in the surfaces improves linearly with the detector dimensions. And last, but not least, improving the performance of the topological signature. The combination of lower radioactive budget and economy of scale may yield a reduction factor of 3 in the overall background rate. Thus, at least another factor 3 will need to come from the topological signature.

\subsection{Improving the topological signature}

A detailed study using DNNS (deep neural networks) [15] has shown that the performance of the topological signature depends critically of the size of the voxelization used to reconstruct the tracks in the detector. This is clearly illustrated in figure 7, where one can see that the tracks reconstructed using $2 \times 2 \times 2 \mathrm{~mm}^{3}$ voxels are sharper than the tracks reconstructed with the NEXT100 standard voxelization $\left(10 \times 10 \times 5 \mathrm{~mm}^{3}\right.$ voxels $)$ which look much coarser. This coarseness results in loss of resolution to identify the blobs at the track extrema as well as potential floating blobs near the track (which are the tell-tale signature of a photoelectric interaction). The DNN analysis shows that a factor 4 improvement in the background rejection rate can be achieved using the finer voxelization.

The voxelization used by the reconstruction algorithms, on the other hand, is fully correlated with the diffusion of the ionization cloud. The large intrinsic diffusion in pure xenon (of the order of $10 \mathrm{~mm} / \sqrt{\mathrm{m}}$ for the transverse diffusion) results in a blurred track, thus the need of a large vox- 
elization and the loss of crucial details. It is possible, however, to reduce the diffusion by a factor five, to some $2 \mathrm{~mm} / \sqrt{\mathrm{m}}$, by adding a suitable quencher gas capable of reducing the diffusion while keeping the primary scintillation and not degrading the energy resolution. Recent studies within the NEXT collaboration suggest that this can be achieved with a mixture $0.85-0.15$ of xenon-helium.

\subsection{Replacing PMTs with SiPMs}

On the other hand, the use of PMTs is not practical for helium mixtures, given the fact that $\mathrm{He}$ diffuses easily into the PMTs, ruining their performance. Furthermore, PMTs cannot be operated at high pressure and are the leading source in the NEXT-100 radioactive budget. The obvious replacement for PMTs are large-area SiPMs. Many companies manufacture SiPMs of up to $10 \times$ $10 \mathrm{~mm}^{2}$. SiPMs operate very well at high pressure and low temperature. In fact, lowering the temperature by 10 degrees celsius reduces the dark current of SiPMs by a factor 2 .

The two main difficulties that have to be solved are: a) to manufacture ultra-low background SiPMs; b) to reduce the dark current to tolerable levels.

In fact, the NEXT collaboration is leading this field, after the successful development of radiopure SiPMs for the tracking plane of the NEW detector [16] and the procedures and methodology are already in place. Dark current, on the other hand, must be reduced in order to detect low-energy X rays, used for calibrations in NEXT. Typically the primary scintillation (S1) of those $\mathrm{X}$-rays (e.g, Krypton lines) is of the order of 10 photoelectrons (pes), integrated over 500 microseconds. SiPMs today have a dark current of about $10 \mathrm{MHz}$ per square centimetre, or $10^{7}$ counts per detection unit in the energy plane. This translates into a rate of about 5 counts in $500 \mathrm{~ns}$. In order to detect comfortably the $\mathrm{Kr} \mathrm{S1}$, therefore, dark current must be reduced by at least one order of magnitude. Operating the SiPMs at $-20 \mathrm{C}^{\circ}$ results in a reduction of dark current of 16 and thus an acceptable dark rate of 0.3 counts.

\subsubsection{NAUSICAA}
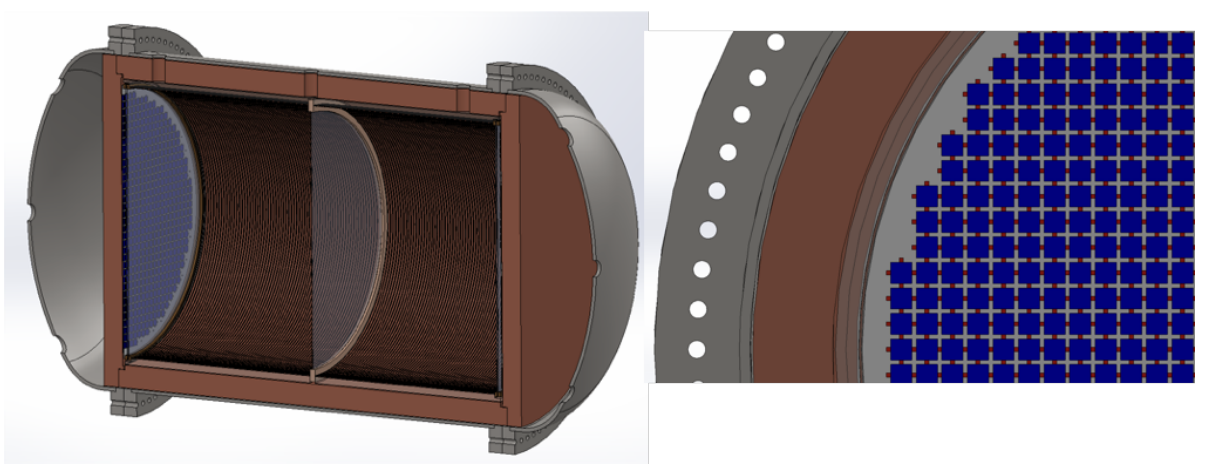

Figure 8: The modules of the NAUSICAA array will be symmetric TPCs with sensor planes performing simultaneously the energy and tracking.

The proposed third stage of the NEXT experiment is NAUSICAA ${ }^{2}$, an array of several modules of 0.5 ton mass, hosted in different underground laboratories (among the possibilities would

\footnotetext{
${ }^{2}$ Next Array apparatus with Improved CApAbilities
} 
be: LSC in Spain, LNGS in Italy, Surf in the USA, and Snolab in Canada). The construction of several modules of (relatively) modest size permits a (relatively) straight forward extrapolation from NEXT-100 and offers a number of advantages such as a better control of instrumental and environmental systematic errors.

The NAUSICAA modules (figure 8) could be symmetric TPCs, with a diameter of $1.4 \mathrm{~m}$ and a drift length of $1.5 \mathrm{~m}$ (thus a total length of $3 \mathrm{~m}$ ). They could operate at a pressure of $20 \mathrm{bar}$. The fiducial mass of each module would be $\sim 550 \mathrm{~kg}$. The two readout planes will be identical, and will be made of SiPMs. Energy (and $\left.t_{0}\right)$ will be measured with large SiPMs $\left(10 \times 10 \mathrm{~mm}^{2}\right)$, arranged at a pitch of $11 \mathrm{~mm}$ while the tracking will be made with small SiPMs $\left(1 \times 1 \mathrm{~mm}^{2}\right)$, also arranged at a pitch of $11 \mathrm{~mm}$. This requires about 13,000 large SiPMs and 13,000 small SiPMs per tracking plane. The energy coverage with this arrangement is much larger than using PMTs (more than $80 \%$ ).

The principle of operation is as follows. Consider a symmetric TPC with two halves A and B. Suppose than an event is produced in the half-sector A. Charge will drift to the EL region of A where it will be amplified and EL light will be produced isotropically. Light moving forward will reach the SiPMs in plane A. Only the information of the small SiPMs in plane A will be used (to determine the xy coordinates of the event), since the large SiPMs directly receiving the EL light will saturate. However, the light moving backwards will be collected by the SiPMs in plane B and integrated, providing a good measurement of the energy of the event. The primary scintillation will be read by both planes.

The NAUSICAA modules will be $50 \%$ larger in diameter and $50 \%$ longer in drift length (per half TPC) than NEXT-100, and thus the technical extrapolation appears feasible. The total number of SiPMs per module will be in the vicinity of $50 \mathrm{k}$. This is to be compared with the roughly 8,000 small SiPMs in NEXT-100. Furthermore, both NEW and NEXT-100 extract the signals from the SiPMs to front-end electronics located outside the chamber using flat cables. Instead, the large number of channels foreseen in NAUSICAA imposes that in-chamber, ASICs electronics will be used.

\subsubsection{Expected sensitivity of NAUSICAA}

A reduction of 3.5 in the background rate is expected for the NAUSICAA modules. This is achieved by a combination of the economy of scale (the detector longitudinal dimensions increase by a factor of 1.5 and so does, roughly, the signal over noise) and a reduction of at least a factor 2 in the radioactive budget, by: a) elimination of the PMTs (the leading source of background); b) fabricating less radioactive SiPM boards (the SiPM circuits, made of multi-layer kapton, are the second leading source of background and the origin of the radioactivity has been traced back to the glue between the layers), c) adding $1 \mathrm{~cm}$ of electroformed copper to further suppress the background arising from the shield. The use of a low diffusion Xe-He mixture, on the other hand, together with advanced compute vision techniques for the reconstruction of the electron tracks should result in an improvement of the topological signature by another factor 3-4. Thus, one order of magnitude background suppression appears possible, and therefore each NAUSICAA module would record less than 0.5 counts per ton and year in the ROI. This would allow NAUSICAA to fully cover the $\mathrm{IH}$. 


\section{Conclusion}

The first radiopure detector in the NEXT series, NEW, has started operations at the Canfranc Underground Laboratory, and the initial results confirm the excellent energy resolution and effective topological signature of the technology. The second state of the project, the NEXT-100 detector, which search for $\beta \beta 0 v$ events starting in 2019. The third stage will be the NAUSICAA array, capable of fully exploring the inverse hierarchy.

\section{References}

[1] J. J. Gomez-Cadenas, J. Martin-Albo, M. Mezzetto, F. Monrabal, and M. Sorel, "The Search for neutrinoless double beta decay," Riv. Nuovo Cim., vol. 35, pp. 29-98, 2012.

[2] O. Cremonesi, "Experimental searches of neutrinoless double beta decay," 2012.

[3] S. Dell'Oro, S. Marcocci, M. Viel, and F. Vissani, "Neutrinoless double beta decay: 2015 review," Adv. High Energy Phys., vol. 2016, p. 2162659, 2016.

[4] M. Agostini et al., "Background free search for neutrinoless double beta decay with GERDA Phase II," 2017. [Nature544,47(2017)].

[5] J. B. Albert et al., "Search for Neutrinoless Double-Beta Decay with the Upgraded EXO-200 Detector," 2017.

[6] A. Gando et al., "Limit on Neutrinoless $\beta \beta$ Decay of ${ }^{136}$ Xe from the First Phase of KamLAND-Zen and Comparison with the Positive Claim in ${ }^{76}$ Ge," Phys. Rev. Lett., vol. 110, no. 6, p. 062502, 2013.

[7] F. Alessandria et al., "Sensitivity of CUORE to Neutrinoless Double-Beta Decay," 2011.

[8] D. Nygren, "High-pressure xenon gas electroluminescent TPC for Onu beta beta-decay search," Nucl.Instrum.Meth., vol. A603, pp. 337-348, 2009.

[9] V. Alvarez et al., "NEXT-100 Technical Design Report (TDR): Executive Summary,” JINST, vol. 7, p. T06001, 2012.

[10] J. Gomez-Cadenas et al., "Present status and future perspectives of the NEXT experiment," 2013.

[11] V. Alvarez et al., "Near-Intrinsic Energy Resolution for 30 to $662 \mathrm{keV} \mathrm{Gamma} \mathrm{Rays} \mathrm{in} \mathrm{a} \mathrm{High}$ Pressure Xenon Electroluminescent TPC," Nucl. Instrum. Meth., vol. A708, pp. 101-114, 2012.

[12] P. Ferrario et al., "First proof of topological signature in the high pressure xenon gas TPC with electroluminescence amplification for the NEXT experiment," JHEP, vol. 01, p. 104, 2016.

[13] B. Jones, A. McDonald, and D. Nygren, "Single molecule fluorescence imaging as a technique for barium tagging in neutrinoless double beta decay," Journal of Instrumentation, vol. 11, no. 12, p. P12011, 2016.

[14] J. Martín-Albo et al., "Sensitivity of NEXT-100 to Neutrinoless Double Beta Decay,” JHEP, vol. 05, p. $159,2016$.

[15] J. Renner et al., "Background rejection in NEXT using deep neural networks," JINST, vol. 12, no. 01, p. T01004, 2017.

[16] S. Cebrián et al., "Radiopurity assessment of the tracking readout for the NEXT double beta decay experiment," JINST, vol. 10, no. 05, p. P05006, 2015. 